\title{
SmallSats Rational Design Technique
}

\author{
Nickolay Zosimovych, \\ $\mathrm{Ph} \mathrm{D}$ in Aerospace Engineering, \\ Professor \\ Department of Mechatronics Engineering, \\ Shantou University, \\ STU, Guangdong, \\ Shantou, China,
}

\begin{abstract}
The SmallSat design process is comprised of choice of its trajectory, determination of its components and main parameters of its systems, development of external and internal layouts, determination of the number of satellite-born antennas and their main characteristics. This paper will focus on estimating a concept and physical relationships in the design process, and on the rational design algorithm version. In terms of specialization of engineering works during SmallSats development, was formulated concept of the design process and established physical relationships to find some optimal design solution about compatibility of basic parameters and characteristics.
\end{abstract}

Keywords - Spacecraft, Smallsat, Design, Accuracy, Configuration, Shape, Limitation, Weight, Equipment, System

\section{INTRODUCTION}

Small spacecraft (SmallSats) focus on spacecraft with mass less than 180 kilograms and about the size of a large kitchen fridge.

The SmallSat design process is comprised of choice of its trajectory, determination of its components and main parameters of its systems, development of external and internal layouts, determination of the number of satellite-borne antennas and their main characteristics, making programs: general one and for separate sessions [1]. Furthermore, since it is not possible to determine any basic parameters for the systems and the requirements for the control system, and to pro-gram the work without understanding the behavior of the individual systems and their interaction, these problems must be solved in the design process.

\section{CONCEPT OF THE DESIGN PROCESS}

In terms of specialization of engineering works in the process of SmallSats development, design and calculation works, development of logical and electric diagrams and development of computation programs, modelling and computer analyses shall be done. The calculation and the modelling process include among others [2-5]:

1) design and strength checking calculations;

2) mass calculations, momentum of inertia calculations, the canter of mass position and positions of the main inertia axes;

3) thermal calculations;

4) calculations of internal and external disturbing moments influencing SmallSat;
5) gas environment calculations for hermetic compartments;

6) estimation of probability of meteorite impact and erosion of external surfaces, determining whether special protection measures (additional screens, thicker shells, more resistant coatings, etc.) should be applied;

7) estimation of radiation exposure for devices, glass, coatings and structural non-metallic elements;

8) dynamic analysis purposed to determine requirements or to check stiffness of the structure to eliminate mutual undesirable influence of mechanical and mechatronic devices and systems, and operation of the orientation system;

9) ballistic design;

10) power supply system calculations, orientation system and other system calculations.

11) If we bind the design process with the development stages typical for any product $[6,7]$, then this process should cover development and agreement of the technical specification for the SmallSat concerned, development of draft proposal, conceptual and technical design (Fig 1).

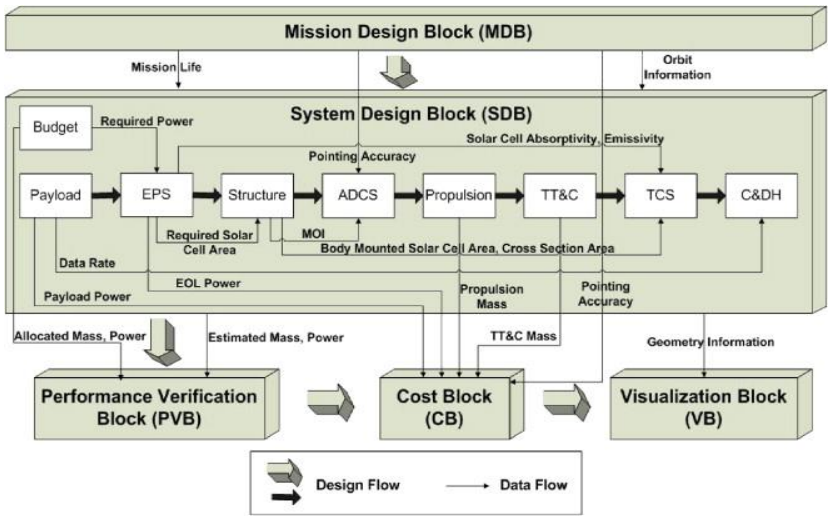

Fig. 1. System Engineering Design Tool [8].

It is obvious that in the process of SmallSat design the basic parameters of separate systems, trajectory characteristics, operation program and the spacecraft design should be brought into line [7].

The external configuration of the SmallSat and optical characteristics of external surfaces determine the characteristics of forces and moments of the light pressure; in some cases, moments of light pressure can be used as useful moments helping to adjust consumption of propellant or electric power for orientation of the SmallSat [9-13]. 


\section{PHYSICAL RELATIONSHIPS IN THE DESIGN PROCESS}

The study of physical relationships in the design process is necessary, first, to find some optimal design solutions about compatibility of basic parameters and characteristics of SmallSat.

The first task is to be certainly solved in the development of any project $[14,15]$. Taking into consideration relatively low cost of modern SmallSats, and the current methods of test and control, it is difficult to imagine that the parameters of any systems could be incompatible in an orbiting SmallSat, or that its design could not provide for the operation of its devices. Such cases are extremely rare. Substantially the alignment of the basic system parameters with each other, and with the characteristics of the trajectory and the design, is the design itself in the usual sense of the word [6].

The second of the tasks set is the search for optimal combinations of parameters and characteristics. It is much more difficult than the first one and is not al-ways solved. This is mainly due to the complexity of studies of this sort [14-16].

This complexity is aggravated by the fact that the external and internal configurations significantly influence the system parameters, mass and other characteristics of SmallSat.

The variety of SmallSats shapes due to minimum external shape limitations for most of them significantly complicates the formalization process enabling to find the best external configuration. And the technology CubeSat is the most effective form for SmallSats today [1].

To avoid a random choice, sometimes development of the components is assigned to different specialists with the following choice of the best option. But also, in this case the choice of the right option is often done based on intuition of the project manager, and therefore, personal preferences, a wish to simplify the analysis and the following works, and other considerations are sub-consciously involved in this choice, which does not always result in the best option or an option close to the best one.

At the same time, inadequate choice of the external configuration can lead to higher values of moments of inertia for SmallSat, increased weight of the on-board cable network, deterioration of characteristics of airborne antennas, complication of technology, etc.

To enable rational design, it is necessary to establish some criteria, which extreme values must be a goal in searching a combination of parameters and characteristics of SmallSat. These criteria are to be determined by the tasks set for a specific spacecraft, or a technical specification for the spacecraft, determining its purpose and operating conditions.

Due to the wide variety of modern SmallSats, it is impossible to enumerate all the criteria that their developers may encounter (Fig. 2).

For some SmallSats the weight of the scientific equipment, which may be installed on the spacecraft, can be a criterion. In the simplest case the trajectory and the orbit injection launch vehicle shall be set. They shall determine the overall weight $M_{0}$ of the spacecraft to be injected on the specified trajectory.
In this case the weight of the equipment $M_{S c}$ will be

$$
M_{S c}=M_{0}-M_{S S},
$$

where $M_{0}-$ is the total weight of the service systems, frame and on-board cable network necessary to ensure the operation of the spacecraft.

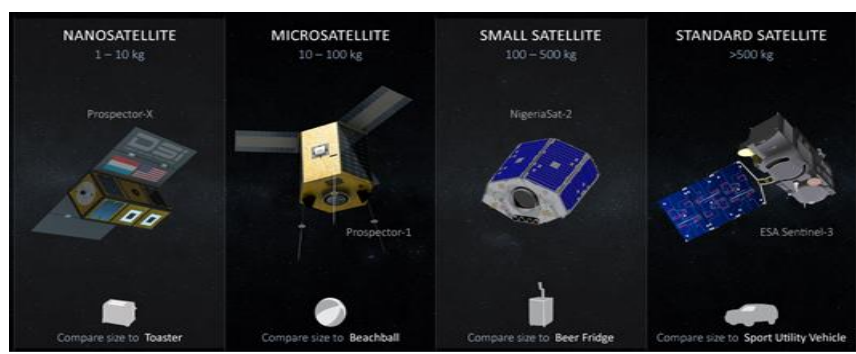

Fig. 2. Satellite Comparison (Deep Space Industries)

Thus, in the simplest case under consideration, when the trajectory, or rather narrow range of trajectories and the launcher are specified, the task of rational design is reduced to minimization of the total weight of service systems, the frame and the on-board cable network when it comes to mathematics. In this case, the initial weight of the vehicle $M_{0}$ can be considered a design value.

Here we proceed from the assumption that the larger is the weight of scientific equipment, the higher is the scientific value of the spacecraft [17]. This assumption seems to be true provided a careful and informed selection of scientific tasks was done.

The described above approach to rational design does not depend on the weight of scientific equipment when the minimum of the total weight of the service systems is to be found $[14,15]$. This approach has very limited application, as in most cases the weight of temperature control devices, electronics, the power supply system and the orientation system depend on the weight of scientific equipment, its purpose and operation program (Fig. 3).

For the cases when the value $M_{S S}$ in the expression (1) cannot be considered independent of the value $M_{S c}$, sometimes it is possible to write the following [17]:

$$
M_{S c}+f_{S S} M_{S c}=M_{0}-M_{S c}^{0},
$$

instead of the specified expression, where $M_{S c}^{0}-$ is the total weight of the service systems and the frame independent of the weight of the scientific equipment; $f_{S S} M_{S c}-$ is an additional weight of the service systems and the frame, necessary for operation of the scientific equipment depending on its weight, composition and operation program. 


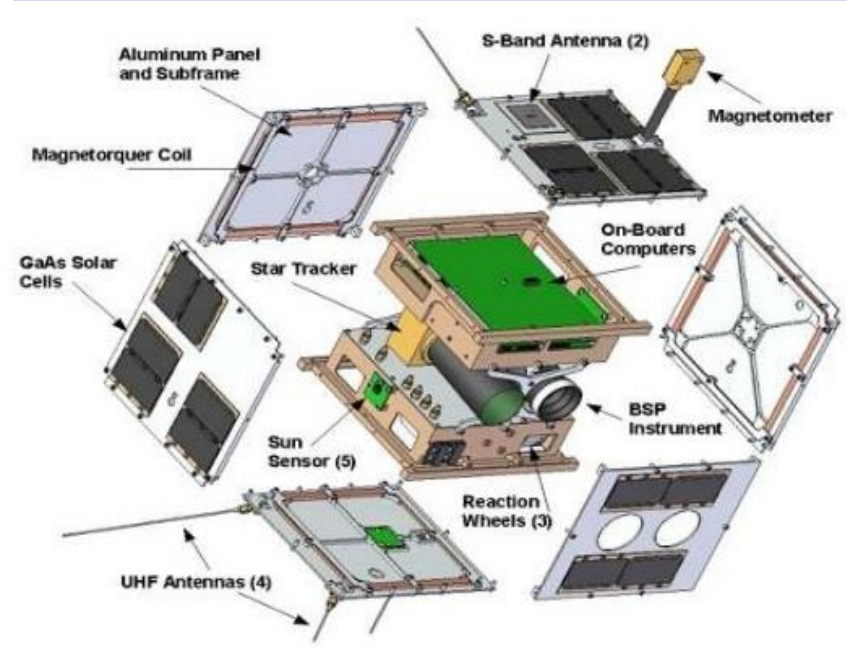

Fig. 3. Basic Components of SmallSat

Various methods of solving the problem of rational design are possible here. For example, we can search a minimum value $M_{S c}^{0}$ in the expression (2), and divide the resulting value $M_{S c}+f_{S S} M_{S c}$ into the weight of the scientific equipment and an additional weight of service systems and frame $f_{S S} M_{S c}$. If the function $f_{S S} M_{S c}$ is quite simple, the expression (2) can be solved relative to the value $M_{S c}$, i.e. find the expression

$$
M_{S c}=F\left(M_{0}, M_{S S}^{0}\right) .
$$

In this case, we can search for the maximum value directly $M_{S c}$.

The above method of problem solving may be not strict enough in some cases. The matter is that that the function $f_{S S} M_{S c}$ characterizing the increase in the weight of the service systems and the frame necessary for the successful functioning of scientific equipment depends, as a rule, on the parameters of the temperature control system, the orientation system, and the power supply system (type of power generator and battery type), the frequency range of the radio telemetric system and the configuration of the spacecraft. If it is impossible to specify the above parameters and the configuration prior to the beginning of the computational analysis, it is impossible to use the formula (3) in the rational design, because to obtain the formula, it is necessary to know the exact type of function, $f_{S S} M_{S c}$ and it is presumably determined by yet unknown versions of service systems [18].

If we like a minimum of the value $M_{S S}^{0}$ in the expression (3), i.e. neglect the weight of the scientific equipment, it also can be approximate, as the versions of the service systems disregarded in the design process can provide a smaller value $f_{S S} M_{S c}$ than the chosen versions [17]. In addition, it is possible that the type of function $f_{S S} M_{S c}$ depends on the research program, which in its turn is determined by the value $M_{S c}$.

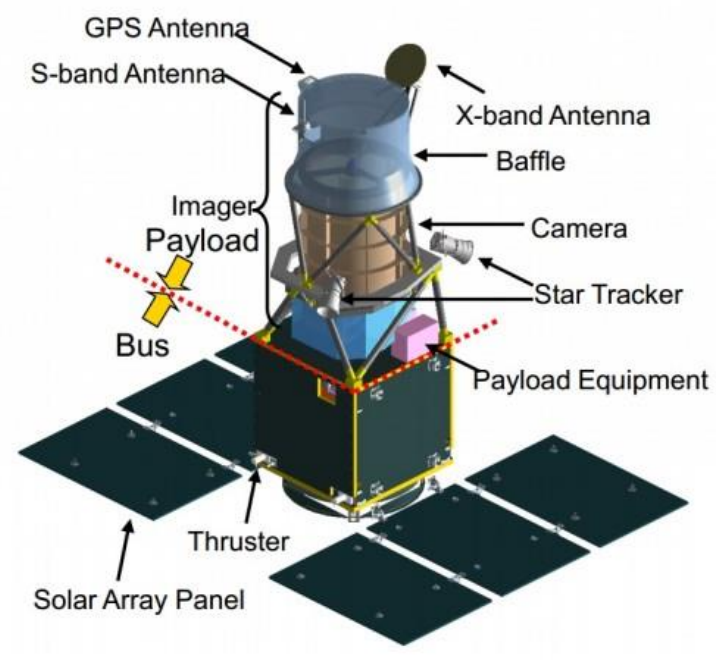

Fig. 4. Satellite ASNARO-1

Another method of solving the problem is more precise, though more painstaking. It supposes determination of the weight of the scientific equipment, its components and the operation program for all the versions of the service systems and configurations of the specified SmallSat provided $M_{0}$. Here each version of the service systems and configuration is basically provided with complete or almost complete development of the project and final adjustment of the basic parameters of all on-board systems and characteristics of SmallSat (Fig. 4). As a rule, rational design in this case should be done by successive approximations. In this case, the expression of the type (3) can always be used to solve some specific problems. For example, for a power supply system consisting of some solar panel and a chemical battery of a type, the function $f_{S S} M_{S c}$ can be easily specified if it is possible to determine the dependence of the average electricity demand for the scientific equipment from its weight [5].

The reliability can be expressed through probability of implementation of the basic task under which here it is necessary to understand operation of scientific equipment according to the specified program within the given time. This time is sometimes called the vehicle operation time, or active existence time [17].

For numerical estimation, the reliability shall be regarded as a probability of flawless operation within a specified time, the failure being such condition of the on-board systems and devices, which makes impossible further functioning of the scientific equipment. To calculate the probability, it is possible to use the theory of reliability apparatus [3].

If we indicate the probability of flawless operation of the spacecraft within a specified time $t_{0}$ as $B$, we can write

$$
B=B\left\lfloor\left(C_{m, n}\right),\left(T_{i}\right),\left(P_{j}\right), t_{0}\right]
$$


where $\left(C_{m, n}\right)$ - is the finite set of basic parameters of systems; $m$-is the system number; $n-$ is a parameter number; $\left(T_{i}\right)-$ is a parameters set determining the trajectory of the spacecraft; $\left(P_{j}\right)$-parameter set determining the operation program.

Among the many parameters of the system there can be those, which are uniquely determined by the composition and characteristics of scientific equipment, and its operation program. The remaining parameters are free. And their choice is the result of rational design. A similar remark can be made concerning parameters $\left(T_{i}\right)$ and $\left(P_{j}\right)$. For example, if we design an artificial Earth satellite with the specified height of a circular orbit and the specified deviation, the orbit injection time shall be free parameter. This parameter determines the orbit position relative to the Sun and stars and can be selected to ensure maximum reliability of the orientation control system at the beginning of orbital motion, particularly when searching and capturing support landmarks.

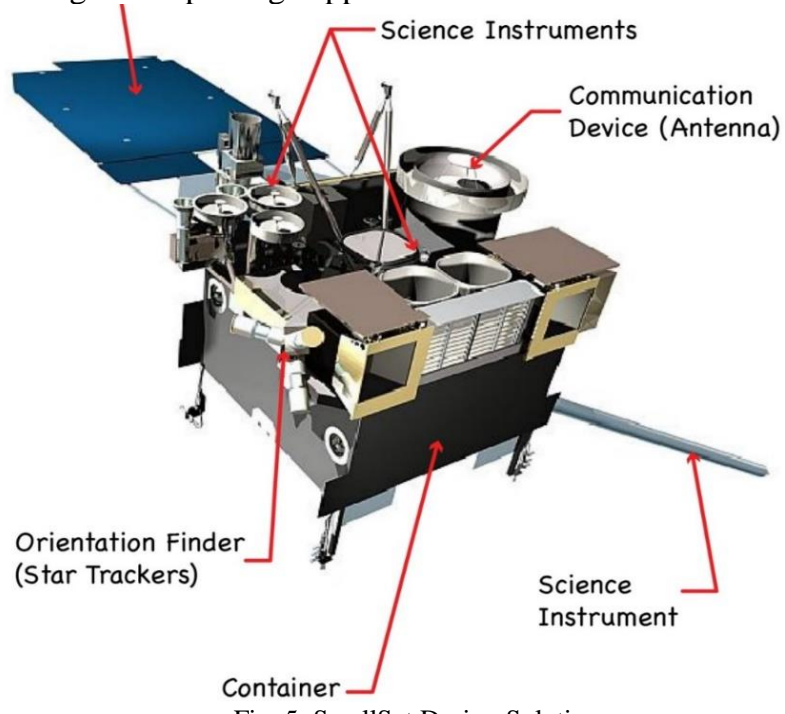

Fig. 5. SmallSat Design Solution

It should be noted that the probability value of flawless operation of the spacecraft is not significant. This value shall be used only as a criterion for analyzing different design solutions. If the technical task sets the value of reliability $B_{0}$, the process of rational design shall consider the condition $B \geq B_{0}$.

Restraints and requirements to the newly developed SmallSat and first its purpose is expressed not only in the form of constants in equations of physical relations [15], but also in presence or absence of the equations and in the form of the equations themselves. This is natural, since the composition and technical meaning of the basic parameters of the systems, trajectory parameters and parameters of the operation program depend on the schemes of the on-board systems, the structure of the operation program and the flight scheme [6]. These parameters are essentially dependent on the purpose of SmallSat and many restrictions and requirements. Hence, the equations of physical relations showing interdependence of all the above parameters [20] depend on the purpose of the satellite, limitations and requirements thereto (Fig. 5).
Besides the availability of partial restraints and requirements narrows the range of basic system parameters, trajectory parameters, operation program and even configuration diagrams considered in the design process. In this case, some equations of physical relationships will not have any solution if there are constants in these equations determining partial restraints and requirements.

It follows from the above that in the process of rational design we must consider equations and inequalities determining physical relationships characteristic for the spacecraft of this purpose or type, and limitations and requirements to it. These expressions will include some constants.

These equations and inequalities shall be written as follows

$$
\Phi_{r}\left[\left(C_{m, n}\right),\left(T_{i}\right),\left(P_{j}\right)\right]\left\{\begin{array}{l}
=0, \\
\geq 0,
\end{array}\right.
$$

where $n=1,2, \ldots, N_{m} ; N_{m}-$ is the number of basic parameters of the $m-$ th system; $m=1,2, \ldots, M ; M-$ is a number of onboard systems; $i=1,2, \ldots, I ; r=1,2, \ldots, R ; j=1,2, \ldots, J$. Using the introduced symbols for all the basic parameters $N_{\Sigma}$ we can write the following expression:

$$
N_{\Sigma}=\sum_{m=1}^{M} N_{m}+I+J
$$

In general, the expressions (5) and (6) may include time.

If all the expressions (5) are equations and $R<N_{\Sigma}$, then the task of seeking for optimal parameter values is confined to finding a constrained extrema of the many variables function. The relations of type (5) are simultaneously the constraint equations.

If some relations (5) are inequalities, the task of seeking the variable $\left(C_{m, n}\right),\left(T_{i}\right)$ and $\left(P_{j}\right)$, minimizing the value $K$, is to be referred to as a task of linear or nonlinear programming depending on the type of functions $K$ and $\Phi$ [18]

The purpose of rational design is to create a project of a vehicle for which the value of the selected criterion is close to the maximum or minimum value. In this case, different configuration diagrams, different orientation schemes and different methods of creating control and corrective forces, should be considered [19]. Depending on the versions of design solutions the functions $K$ and $\Phi$ will change. Consequently, the rational design shall be confined to the investigation of the function in the constraint equations (5) for different versions of the newly designed SmallSat.

Finding the optimal parameter values for one record variant of functions $K$ and $\Phi$ shall be described as a specific task of rational design. This is essentially the task of optimizing some specific version of SmallSat.

In some cases, the analysis of physical relations characteristic for some versions of SmallSat allows to find an optimal combination of some parameters, which simplifies the solution of the rational design problem. Mathematically the above means that it is possible to extract from the system (5) a 
subsystem including only some parameters and find some specific criterion depending on these parameters and not contradicting the general criterion $K$. The tasks of this type can be called specific optimal tasks of SmallSat design.

\section{RATIONAL DESIGN ALGORITHM VERSION}

Before we can provide a variant of the rational design algorithm let's make some assumptions concerning equations and inequalities (5) [18]. Basically, these assumptions specify a class of spacecraft for which they are true and for which we are going to offer an algorithm.

The relations (6) include only equations, inequalities are absent. This is because inequalities arising from the requirements to the spacecraft and from limitations can be replaced by equations for many spacecraft's. If it is required that the initial weight of the spacecraft does not exceed the specified value determined by the trajectory and the launch vehicle, then when analyzing the different versions of SmallSat and identifying its optimal parameters it is possible to accept that the initial mass of the spacecraft $M_{0}$ is equal to the maximum permissible value $M_{0_{\max }}$ minus some allowance i.e.

$$
M_{0}=M_{0_{\max }}\left(1-\frac{\Delta M}{M_{0_{\max }}}\right) .
$$

The relative weight allowance may be accepted within [17] depending on the complexity and novelty of the developed SmallSat and its systems.

Similar reasoning can be given also for the case when the minimum permissible reliability of SmallSat is specified, and in relations (5) we shall accept, that the reliability of the spacecraft is equal to this value with some allowance which can disappear at the stage of detailed design.

If some of the parameters in the equations (5) are timedependent, for example, the weight of a spacecraft or its moments of inertia due to fuel consumption on during correction phase, maneuvers or orientation process, the time may be included as constants obtained during the ballistic design phase. For example, the equations (5) may include members $n_{1}$ and $n_{2}$, where $n-$ is an average fuel consumption per second needed for orientation of the spacecraft. It is one of the varied parameters of the orientation control system depending on the moment of inertia, arms of the driven engines, disturbing moments, etc., $t_{1}$ and $t_{2}-$ constants determining the times of characteristic points within the flight trajectory.

The varied parameters do not include any parameters and characteristics of SmallSat trajectory. Consequently, it is assumed that the choice of the flight scheme, the basic parameters of the trajectory, as well as determination of the requirements to the spacecraft in terms of implementation of the necessary trajectory has been done in advance, before determining the parameters of systems, configuration and the operation program. Such a stage of work, which is called ballistic design, can often be started immediately after receiving technical specifications for the SmallSat. In cases when there is a dependence of the trajectory parameters from the parameters of some systems, and the latter cannot be determined in advance before the complex investigation of the spacecraft parameters, it is necessary to use the method of consecutive approximations. Ballistic design is an independent area of spacecraft design [18].

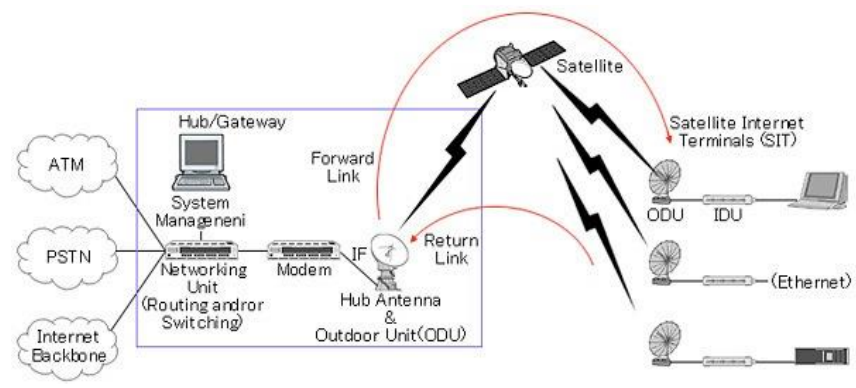

Fig. 6. SmallSat Data Process Flow

The ballistic design shall result in determined trajectory characteristics, the initial weight of the spacecraft, which can be injected in the specified trajectory by the chosen launcher, characteristic speeds, times for corrections and maneuvers, requirements to the control actions for the thrust vector positioning during corrections and maneuvers, and the necessary accuracies, and, in addition, all necessary data for the development of the orientation control system and operation program, such as, for example, the angles between possible optical guides and the times when the spacecraft is in the visual range of ground facilities (Fig. 6).

It should be noted that at the stage of ballistic design may be necessary to solve complex variation problems, multipoint boundary value problem, etc. Some of these tasks are studied in the publication [19].

The number of constraint equations (5) is less than the number of varied parameters. If this assumption is not fulfilled, the task of selecting the optimal parameters cannot be solved, as there are no free parameters to minimize the criterion $K$. Most likely it means that some free parameters have not been revealed, and it is necessary to review the parameters and the type of functions $K$ and $\Phi$.

Let's assume that at some stage of the design process we found a satellite and a program version which met all the requirements and restrictions. This version of the satellite shall be called a reference one. Suppose that it is characterized by parameters $\left(\bar{C}_{m, n}\right)$ and $\left(\bar{P}_{j}\right)$, that we call the source parameters. These parameters will satisfy the equations (5), which consider the physical relations and constraints characteristic for the found SmallSat variant.

Obviously, the combination and technical signification of the SmallSat parameters, and therefore the structure of the expressions (5) and the constants included therein will not change when the parameters within some intervals near the values $\left(\bar{C}_{m, n}\right)$ and $\left(\bar{P}_{j}\right)$ change. We shall introduce the following symbols for the specified intervals of each parameter:

$$
\left.\left.\left\lfloor C_{m, n}^{\prime}, C_{m, n}^{\prime \prime}\right\rfloor\right\rfloor P_{j}^{\prime}, P_{j}^{\prime \prime}\right\rfloor
$$


For convenience, further under the reference version we will understand the version characterized by parameter variation intervals (6).

Optimization of the reference version is limited to search of parameter values $\left(\bar{C}_{m, n}\right)$ and $\left(\bar{P}_{j}\right)$ within intervals (6) and implementation of the maximum or minimum value of the criterion during execution of equations (3), written for the reference version.

It is very important that the experience of creation and operation of similar spacecraft is used in the development of the versions. The qualification of the developers of the reference versions is of paramount importance. However, it should be borne in mind that the newly created SmallSat may not have prototypes. In such cases, a sufficiently wide review of the possible reference versions is required.

The described method of rational design, of course, does not deny the process of intuitive creative thinking. This process reveals itself in assumptions and development of reference versions, as well as in ballistic design.

In the context of the amount of calculations the most complex is the stage of finding optimum parameters and extreme values for the criterion for all reference versions. For each reference variant the problem is to study the function extrema

$$
K\left(x_{1}, x_{2}, \ldots, x_{N_{\Sigma}}\right)
$$

in constraint equations

$$
\Phi_{r}\left(x_{1}, x_{2}, \ldots, x_{N_{\Sigma}}\right)=0
$$

where $r=1,2, \ldots, R ; x_{k}^{\prime} \leq x_{k} \leq x_{k}^{\prime \prime}, k=1,2, \ldots, N_{\Sigma} ; x_{k}^{\prime}, x_{k}^{\prime \prime}$ correspond to the parameter variation intervals boundaries (6).

There are different methods of solving this problem. First, we can try to exclude some parameters $x_{k}$ using equations (8) and to investigate unconditional extrema of the function $K$ of already $N_{\Sigma}-R$ variables.

If it is difficult, i.e. to investigate the extrema of the function

$$
\bar{\Phi}=K+\sum_{r=1}^{R} \lambda_{r} \Phi_{r}
$$

In this case, the required optimal parameters and multipliers $\lambda_{r}$ are to be found from necessary existence conditions of the internal maximum or minimum function $\bar{\Phi}$

$$
\frac{\partial \bar{\Phi}}{\partial x_{k}}=0, k=1,2, \ldots, N_{\Sigma}
$$

and equations (8).
You can try to linearize the functions $K$ and $\Phi$ by developing them, for example, into the Taylor series near the point $\left(\bar{x}_{1}, \bar{x}_{2}, \ldots, \bar{x}_{N_{\Sigma}}\right)$ and leaving only first-order terms about variations of the parameters $\Delta x_{k}=x_{k}-\bar{x}_{k}$.

The validity of such linearization can always be set, knowing that $x_{k}^{\prime} \leq x_{k} \leq x_{k}^{\prime \prime}$, where $x_{k}^{\prime}$ and $x_{k}^{\prime \prime}$ are the limits of the variation intervals for parameter $x_{k}$. If we take a maximum permissible error of the criterion $K$ we shall get

$$
\Delta K=K-\bar{K}=\sum_{k=1}^{N_{\Sigma}} \frac{\partial K}{\partial x} \Delta x_{k}
$$

resulting $\quad$ from $\quad$ linearization,
$\bar{K}=K\left(\bar{x}_{1}, \bar{x}_{2}, \ldots, \bar{x}_{N_{\Sigma}}\right), x_{k}^{\prime}-\bar{x}_{k} \leq \Delta x_{k} \leq x_{k}^{\prime \prime}-\bar{x}_{k}$.

In this case we get a system of linear equations:

$$
\begin{aligned}
& \frac{\partial \Phi_{1}}{\partial x_{1}} \Delta x_{1}+\ldots+\frac{\partial \Phi_{1}}{\partial x_{N_{\Sigma}}} \Delta x_{N_{\Sigma}}=0 \\
& \frac{\partial \Phi_{R}}{\partial x_{1}} \Delta x_{1}+\ldots+\frac{\partial \Phi_{R}}{\partial x_{N_{\Sigma}}} \Delta x_{N_{\Sigma}}=0 .
\end{aligned}
$$

instead of the constraint equations (8).

In the expressions (10) and (11) all the partial derivatives shall be calculated in the point $\left(\bar{x}_{1}, \bar{x}_{2}, \ldots, \bar{x}_{N_{\Sigma}}\right)$. By excluding a part of parameters using equations (11), we shall find a formula for $\Delta K$ as follows

$$
\Delta K=\sum_{l=1}^{N_{\Sigma}-R} e_{l} \Delta x_{l}
$$

This is a hyperplane equation in a multidimensional space $\left(\Delta x_{1}, \Delta x_{2}, \ldots, \Delta x_{N_{\Sigma}-R}, \Delta K\right)$. Therefore, the value $\Delta K$ can take the largest or the smallest value only at the boundaries of the value variation intervals $\Delta x_{l}$.

\section{CONCLUSION}

Thus, here finding of an optimal combination of parameters $\Delta x_{l}$ shall be confined to calculation of the value for all possible variation intervals boundary combinations $\Delta K$ and selection of a combination $\Delta x_{l}$ implementing the maximum or minimum values $\Delta K$.

Please note that the described method is a special case of the linear programming problem. 


\section{REFERENCES}

[1] https://www.nasa.gov/cubesats/ - What are SmallSats and CubeSats? Editor: Elizabeth Mabrouk Feb. 26, 2015.

[2] D.J. Bayley, Design Optimization of Space Launch Vehicles Using a genetic Algorithm, Auburn University Alabama, 2007.

[3] T.T. Furman, Approximate Methods in Engineering Design, vol. 155 Math. in Science and Engineering Edited by Richard Bellman, 1981.

[4] N. Zosimovych, Modeling of Spacecraft Centre Mass Motion Stabilization System, Int. Refereed Journal of Engineering and Science, vol. 6, Issue 4, pp. 34-41.

[5] N. Zosimovych, Space vehicle identification parameters on the basis of an optimum filtration, Int. Conf. on Modern Achievements of the European Science - 2009, vol. 10, Sofia: БялГРАД-БГ/ Technologies, pp 42-46, 2009.

[6] N. Zosimovych, Commercial Launch Vehicle Design, LAP LAMBERT Academic Publishing p. 184, 2016.

[7] И.С. Голубев, А.В. Самарин, Проектирование конструкций летательных аппаратов, М.: Машиностроение, р 512, 1991.

[8] Y.K. Chang, K.Y. Hwang, S.J. Kang, SEDT (System Engineering Design Tool) development and its application to small satellite conceptional design, Acta Astronautica, vol. 61, No 7-8, pp. 676-690.

[9] N.V. Zosimovych, Substantiation of monitoring space system parameters of the Earth, Int. Conf. on Modern Technologies in Aerospace Complex, Zhytomyr State Technological Institute, Sept. 7-9, pp. 170-173, 1997.

[10] Р.Ф. Аппазов, О.Г. Сытин, Методы проектирования траекторий носителей и спутников Земли, М.: Наука, р. 440, 1987.

[11] Б.Ф. Жданюк, Основы статистической обработки траекторных измерений, М.: Машиностроение, p. 384, 1978.
[12] N. Zosimovych, Improving the Spacecraft Center of Mass Stabilization Accuracy, IOSR Journal of Engineering, vol. 7, Issue 6, pp. 7-14, 2017.

[13] N. Zosimovych, Increasing the Accuracy of the Center of Mass Stabilization of Space Probe, Int. Conf. on Новината за Напреднали Наука - 2017, Мау 15-22, vol. 10, София: Бял ГРАД-БГ ООД/Technical Science, pp. 31-37, 2017.

[14] N. Zosimovych, General Technique for Solving Structural and Parametric Optimization of Flying Vehicle Structures, Int. Conf. on Naukowa Przestrzeń Europy - 2017, Apr. 7-15, vol. 11, Poland: Przemyśl Science and Education LTD/Technical Science, pp. 50-63, 2017.

[15] N. Zosimovych, Structural and Parametric Optimization for Flight Vehicle Structures International Scientific and Practical Conference Areas of Scientific Thought - 2016/2017 Dec. 30 2016 - Jan. 7, Vol. 8, UK: Sheffield/ Technical Science Science and Education LTD, pp. 59-68, 2017.

[16] C. Fish, C. Swenson, T. Neilsen, B. Bingham, J. Gunther, E. Stromberg, S. Bur, R. Burt, M. Whitely, DICE Mission Design, Development, and Implementation: Success and Challenges, 26th Annual AIAA/USU Conf. on Small Satellites, USA: SSC12-XI-1, pp. 1-20, 2017.

[17] Ю.Г. Сихарулидзе, Баллистика летательных аппаратов, М.: Наука, р. 351, 1982.

[18] В.К. Абалакин, Е.П. Аксенов, Е.А. Гребеников и др., Справочное руководство по небесной механике и астродинамике, М.: Наука, р. 864, 1976.

[19] Е.В. Тарасов, Алгоритм оптимального проектирования летательного аппарата, М.: Машиностроение, р. 344, 1970.

[20] Г.Ю. Максимов, Теоретические основы разработки космических аппаратов, М.: Наука, р. 320, 1980. 